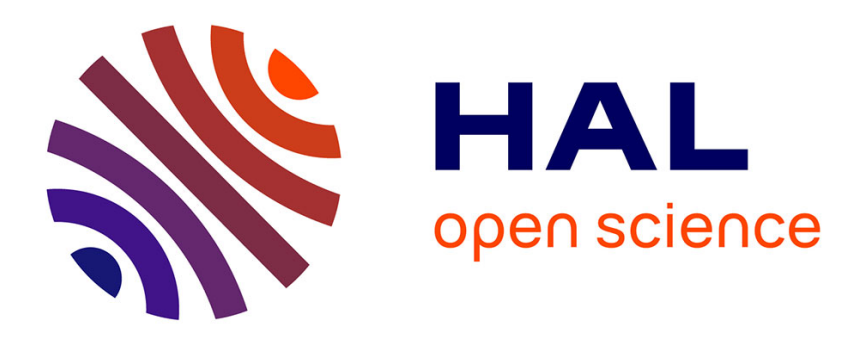

\title{
Characterizations of Total Dual Integrality
}

Edwin O’Shea, András Sebö

\section{- To cite this version:}

Edwin O'Shea, András Sebö. Characterizations of Total Dual Integrality. 12th International IPCO conference, 2007, Ithaca, United States. pp.382-396. hal-00189411

\section{HAL Id: hal-00189411 https://hal.science/hal-00189411}

Submitted on 20 Nov 2007

HAL is a multi-disciplinary open access archive for the deposit and dissemination of scientific research documents, whether they are published or not. The documents may come from teaching and research institutions in France or abroad, or from public or private research centers.
L'archive ouverte pluridisciplinaire HAL, est destinée au dépôt et à la diffusion de documents scientifiques de niveau recherche, publiés ou non, émanant des établissements d'enseignement et de recherche français ou étrangers, des laboratoires publics ou privés. 


\title{
Characterizations of Total Dual Integrality ${ }^{\star}$
}

\author{
Edwin O’Shea ${ }^{1}$ and András Sebö ${ }^{2}$ \\ ${ }^{1}$ Department of Mathematics, University of Kentucky, Lexington, \\ KY 40506-0027, USA \\ oshea@ms.uky.edu \\ ${ }^{2}$ CNRS, Laboratoire G-SCOP, 46, Avenue Félix Viallet, 38000 Grenoble 38031 \\ Grenoble, Cedex 1, France \\ Andras.Sebo@g-scop.inpg.fr
}

\begin{abstract}
In this paper we provide new characterizing properties of TDI systems. A corollary is Sturmfels' theorem relating toric initial ideals generated by square-free monomials to unimodular triangulations. A reformulation of these test-sets to polynomial ideals actually generalizes the existence of square-free monomials to arbitrary TDI systems, providing new relations between integer programming and Gröbner bases of toric ideals. We finally show that stable set polytopes of perfect graphs are characterized by a refined fan that is a triangulation consisting only of unimodular cones, a fact that endows the Weak Perfect Graph Theorem with a computationally advantageous geometric feature. Three ways of implementing the results are described and some experience about one of these is reported.
\end{abstract}

\section{Introduction}

Let $A=\left[\mathbf{a}_{1} \mathbf{a}_{2} \cdots \mathbf{a}_{n}\right] \in \mathbb{Z}^{d \times n}$ and assume that $A$ has rank $d$. With an abuse of notation the ordered vector configuration consisting of the columns of $A$ will also be denoted by $A$. For every $\sigma \subseteq[n]:=\{1, \ldots, n\}$ we have the $d \times|\sigma|$ matrix $A_{\sigma}$ given by the columns of $A$ indexed by $\sigma$. Let cone $(A), \mathbb{Z} A$ and $\mathbb{N} A$ denote the non-negative real, integer and non-negative integer span of $A$ respectively and assume that $\mathbb{Z} A=\mathbb{Z}^{d}$.

Fixing $\mathbf{c} \in \mathbb{R}^{n}$, for each $\mathbf{b} \in \mathbb{R}^{d}$ the linear program (or primal program) $\mathrm{LP}_{A, \mathbf{c}}(\mathbf{b})$ and its dual program $\mathrm{DP}_{A, \mathbf{c}}(\mathbf{b})$ are defined by

$$
\operatorname{LP}_{A, \mathbf{c}}(\mathbf{b}):=\operatorname{minimize}\{\mathbf{c} \cdot \mathbf{x}: A \mathbf{x}=\mathbf{b}, \mathbf{x} \geq \mathbf{0}\}
$$

and $\operatorname{DP}_{A, \mathbf{c}}(\mathbf{b}):=\operatorname{maximize}\{\mathbf{y} \cdot \mathbf{b}: \mathbf{y} A \leq \mathbf{c}\}$. Let $P_{\mathbf{b}}$ and $Q_{\mathbf{c}}$ denote the feasible regions of $\mathrm{LP}_{A, \mathbf{c}}(\mathbf{b})$ and $\mathrm{DP}_{A, \mathbf{c}}(\mathbf{b})$ respectively. Note that the linear program $\operatorname{LP}_{A, \mathbf{c}}(\mathbf{b})$ is feasible if and only if $\mathbf{b} \in \operatorname{cone}(A)$. We refer to Schrijver 21] for basic terminology and facts about linear programming.

* The first author was supported by a Fulbright grant and by NSF grants DMS9983797 and DMS-0401047. The research of the second author was supported by the "Marie Curie Training Network" ADONET of the European Community. 
The integer program is defined as

$$
\operatorname{IP}_{A, \mathbf{c}}(\mathbf{b}):=\operatorname{minimize}\left\{\mathbf{c} \cdot \mathbf{x}: A \mathbf{x}=\mathbf{b}, \mathbf{x} \in \mathbb{N}^{n}\right\} .
$$

We say that $\mathbf{c} \in \mathbb{R}^{n}$ is generic for $A$ if the integer program $\operatorname{IP}_{A, \mathbf{c}}(\mathbf{b})$ has a unique optimal solution for all $\mathbf{b} \in \mathbb{N} A$. In this case, each linear program $\operatorname{LP}_{A, \mathbf{c}}(\mathbf{b})$ also has a unique optimal solution for all $\mathbf{b} \in \operatorname{cone}(A)$ but the converse is not true in general. (However, for TDI systems the two are equivalent.)

The system $\mathbf{y} A \leq \mathbf{c}$ is totally dual integral (TDI) if $\mathrm{LP}_{A, \mathbf{c}}(\mathbf{b})$ has an integer optimal solution $\mathbf{x} \in \mathbb{N}^{n}$ for each $\mathbf{b} \in \operatorname{cone}(A) \cap \mathbb{Z}^{d}$. In other words, the system $\mathbf{y} A \leq \mathbf{c}$ is TDI exactly if the optima of $\operatorname{LP}_{A, \mathbf{c}}(\mathbf{b})$ and of $\operatorname{IP}_{A, \mathbf{c}}(\mathbf{b})$ coincide for all $\mathbf{b} \in \operatorname{cone}(A) \cap \mathbb{Z}^{d}$. This is a slight twist of notation when compared to habits in combinatorial optimization: we defined the TDI property for the dual problem. We do this in order to be in accordance with notations in computational algebra.

Totally dual integral (TDI) systems of linear inequalities play a central role in combinatorial optimization. The recognition of TDI systems and the task of efficiently solving integer linear programs constrained by TDI systems of inequalities and their duals are among the main challenges of the field. This problem is open even for generic systems (Problem 1). Recent graph theory results of Chudnovsky, Cornuéjols, Xinming and Vušković [7] allows one to recognize TDI systems with $0-1$ coefficient matrices $A$ and right hand sides $\mathbf{b}$. However, solving the corresponding dual pair of integer linear programs (including the coloration of perfect graphs) in polynomial time with combinatorial algorithms remains open even in this special case.

In Section 2, new characterizing properties of TDI systems are provided. These properties involve tools from both combinatorial optimization and computational algebra. Section 3 specializes these results to integral set packing polytopes. Finally, Section 4 will exhibit the utility of the computational algebraic tools in recognizing TDI systems.

If $A$ is a matrix whose first $d \times(n-d)$ submatrix is a $0-1$ matrix and whose last $d \times d$ submatrix is $-I_{d}$, and $\mathbf{c}$ is all 1 except for the last $d$ coordinates which are 0 , then $\mathrm{DP}_{A, \mathbf{c}}(\mathbf{b})$ is called a set packing problem, and $Q_{\mathbf{c}}$ a set packing polytope. We will show that if the set packing polytope is integral then the lexicographic perturbation technique of linear programming can be used to make the set packing polytope non-degenerate while keeping TDI-ness. This means that the normal fan of the set packing polytope has a refinement which is a unimodular triangulation, and this does not hold for TDI systems in general.

The remainder of this introduction is devoted to providing some background.

A collection of subsets $\left\{\sigma_{1}, \ldots, \sigma_{t}\right\}$ of $[n]$ will be called a regular subdivision of $A$ if there exists $\mathbf{c} \in \mathbb{R}^{n}$, and $\mathbf{z}_{1}, \ldots, \mathbf{z}_{t} \in \mathbb{R}^{d}$, such that $\mathbf{z}_{i} \cdot \mathbf{a}_{j}=c_{j}$ for all $j \in \sigma_{i}$ and $\mathbf{z}_{i} \cdot \mathbf{a}_{j}<c_{j}$ for all $j \notin \sigma_{i}$. The sets $\sigma_{1}, \ldots, \sigma_{t}$ are called the cells of the regular subdivision and the regular subdivision is denoted by $\Delta_{\mathbf{c}}(A)=\left\{\sigma_{1}, \ldots, \sigma_{t}\right\}$ or simply $\Delta_{\mathbf{c}}$ when $A$ is unambiguous.

Equivalently, regular subdivisions are simply capturing complementary slackness from linear programming. Namely, a feasible solution to $\mathrm{LP}_{A, \mathbf{c}}(\mathbf{b})$ is optimal 
if and only if the support of the feasible solution is a subset of some cell of $\Delta_{\mathbf{c}}$. Geometrically, $\Delta_{\mathbf{c}}$ can be thought of as a partition of $\operatorname{cone}(A)$ by the inclusionwise maximal ones among the cones cone $\left(A_{\sigma_{1}}\right), \ldots$, cone $\left(A_{\sigma_{t}}\right)$; each such cone is generated by the normal vectors of defining inequalities of faces of $Q_{\mathbf{c}}$, each maximal cell indexes the set of normal vectors of defining inequalities of a vertex (or minimal face) of $Q_{\mathbf{c}}$. So the regular subdivision $\Delta_{\mathbf{c}}$ is geometrically realized as the normal fan of $Q_{\mathbf{c}}$.

A regular subdivision of $A$ is called a triangulation if the columns of each $A_{\sigma_{i}}$ are linearly independent for all $i=1, \ldots, t$. Note that a regular subdivision $\Delta_{\mathbf{c}}$ is a triangulation if and only if every vertex is contained in exactly $d$ facets; that is, the polyhedron $Q_{\mathbf{c}}$ is simple, or, non-degenerate. A triangulation $\Delta_{\mathbf{c}}$ is called unimodular if $\operatorname{det}\left(\sigma_{i}\right)= \pm 1$ for each maximal cell of $\Delta_{\mathbf{c}}$. The refinement of a subdivision $\Delta_{\mathbf{c}}$ of $A$ is another subdivision $\Delta_{\mathbf{c}^{\prime}}$ of $A$ so that each cell of $\Delta_{\mathbf{c}^{\prime}}$ is contained in some cell of $\Delta_{\mathbf{c}}$. A vector configuration $B \subset \mathbb{Z}^{d}$ is a Hilbert basis if $\mathbb{N} B=\operatorname{cone}(B) \cap \mathbb{Z}^{d}$. Note that if for some $\mathbf{c} \in \mathbb{R}^{n} \Delta_{\mathbf{c}}$ is a unimodular triangulation of $A$ then Cramer's rule implies that $A$ itself is a Hilbert basis.

A simple but helpful characterization of the TDI property in terms of the Hilbert basis property of regular subdivisions has been provided by Schrijver [21]. We prove another elementary characterization in Section 2 in terms of test-sets:

Let $\operatorname{IP}_{A, \mathbf{c}}:=\left\{\operatorname{IP}_{A, \mathbf{c}}(\mathbf{b}): \mathbf{b} \in \mathbb{N} A\right\}$ denote the family of integer programs $\mathrm{IP}_{A, \mathbf{c}}(\mathbf{b})$ having a feasible solution. Informally, a test set for the family of integer programs $\mathrm{IP}_{A, \mathbf{c}}$ is a finite collection of integer vectors, called test vectors, with the property that any non-optimal feasible solution can be improved (in objective value) by subtracting a test vector from it. Test sets for the family of integer programs $\operatorname{IP}_{A, \mathbf{c}}$ were first introduced by Graver [13.

Theorem 1 (one of the equivalences). A system of linear inequalities is TDI if and only if its coefficient vectors form a Hilbert basis, and there exists a test set for $\mathrm{IP}_{A, \mathbf{c}}$ where all test vectors have positive entries equal to 1 , and a linearly independent positive support 1

This simple result has the virtue of presenting a not too big test-set: there is at most one test-vector for each at most $d$ element subset of $\{1, \ldots, n\}$, so the number of test-vectors is $O\left(n^{d}\right)$. This will allow to deduce shortly Cook, Lovász and Schrijver's result on testing for TDI in fix dimension, providing a short proof for this result.

It also has the other virtue that it has a nice and useful reformulation to polynomial ideals. This reformulation generalizes a well-known algebraic result proved by Sturmfels [26, Corollary 8.9] relating toric initial ideals to unimodular triangulations. The basic connections between integer programming and

\footnotetext{
${ }^{1}$ In oral and electronic communication the condition on test-sets was replaced by the following still equivalent condition: "A system of linear inequalities is TDI if and only if the coefficient vectors form a Hilbert basis, and there exists an integer dual solution for objective functions that are sums of linearly independent coefficient vectors", implying TDI test in fix dimension [5, in practically all interesting cases. This is just another wording of Applegate, Cook and McCormick's Theorem 2 (Operations Research Letters 10 (1991) 37-41), as we learnt from several colleagues.
} 
computational algebra was initiated by Conti and Traverso [3] and studied by Sturmfels and Thomas, Weismantel and Ziegler and further explained from various viewpoints in 26, 25, 27] and 28. Knowledge of this algebraic viewpoint will not be assumed and a useful part will be described in Section 2

In Section 3 we show that the converse of the following fact (explained at the end of Section 2) holds for normal fans of integral set packing polytopes: if $\mathbf{c}, \mathbf{c}^{\prime} \in \mathbb{R}^{n}$ are such that $\Delta_{\mathbf{c}^{\prime}}$ is a refinement of $\Delta_{\mathbf{c}}$, where $\Delta_{\mathbf{c}^{\prime}}$ is a unimodular triangulation, then $\mathbf{y} A \leq \mathbf{c}$ is TDI. In general, the converse does not hold. Thus Schrijver's above mentioned result cannot necessarily be strengthened by asserting a unimodular refinement of $A$. In general, the most that is known in this direction is the existence of just one full dimensional subset of the columns of $A$ which is unimodular 11. Not even a "unimodular covering" of a Hilbert basis may be possible [1. However, the converse does hold for normal fans of integral set packing polytopes. More precisely, the main result of Section 3 is the following:

Theorem 2, Given a set-packing problem defined by $A$ and c, $Q_{\mathbf{c}}$ has integer vertices if and only if there exists $\mathbf{c}^{\prime}$ such that $\Delta_{\mathbf{c}^{\prime}}$ is a refinement of the normal fan $\Delta_{\mathbf{c}}$ of $Q_{\mathbf{c}}$, where $\Delta_{\mathbf{c}^{\prime}}$ is a unimodular triangulation.

The proof relies on the basic idea of Fulkerson's famous "pluperfect graph theorem" 12 stating that the integrality of such polyhedra implies their total dual integrality in a very simple "greedy" way. Chandrasekaran and Tamir [2] and Cook, Fonlupt and Schrijver [4] exploited Fulkerson's method by pointing out its lexicographic or advantageous Caratheodory feature. In [23, §4] it is noticed with the same method that the active rows of the dual of integral set packing polyhedra (the cells of their normal fan) have a unimodular subdivision, which can be rephrased as follows: the normal fan of integral set packing polyhedra has a unimodular refinement. However, the proof of the regularity of such a refinement appears for the first time in the present work.

These results offer three methods for recognizing TDI systems, explained and illustrated in Section 4.

\section{TDI Systems}

In this section we provide some new characterizations of TDI systems. We show the equivalence of five properties, three polyhedral (one of them is the TDI property) and two concern polynomial ideals. A third property is also equivalent to these in the generic case.

While the proofs of the equivalences of the three polyhedral properties use merely polyhedral arguments, the last among them - (iii) - has an appealing reformulation into the language of polynomial ideals. Therefore, we start this section by introducing the necessary background on polynomial ideals; namely, 
toric ideals, their initial ideals and Gröbner bases. The characterizations of TDI systems involving polynomial ideals are useful generalizations of known results in computational algebra. See [8] and 26] for further background.

An ideal $I$ in a polynomial $\operatorname{ring} R:=\mathbf{k}\left[x_{1}, \ldots, x_{n}\right]$ is an $R$-vector subspace with the property that $I \cdot R=I$. It was proven by Hilbert that every ideal is finitely generated. That is, given an ideal $I$ there exists a finite set of polynomials $f_{1}, \ldots, f_{t} \in I$ such that for every $f \in I$ there exists $h_{1}, \ldots, h_{t} \in R$ with $f=$ $h_{1} f_{1}+\cdots+h_{t} f_{t}$. We call such a collection $f_{1}, \ldots, f_{t} \in I$ a generating set for the ideal $I$ and denote this by $I=\left\langle f_{1}, \ldots, f_{t}\right\rangle$. For the monomials in $R$ we write $\mathbf{x}^{\mathbf{u}}=x_{1}^{u_{1}} \cdots x_{n}^{u_{n}}$ for the sake of brevity. We call $\mathbf{u}$ the exponent vector of $\mathbf{x}^{\mathbf{u}}$. A monomial $\mathbf{x}^{\mathbf{u}}$ is said to be square-free if $\mathbf{u} \in\{0,1\}^{n}$. An ideal is called a monomial ideal if it has a generating set consisting only of monomials. For any ideal $J$ of $R$, mono $(J)$ denotes the largest monomial ideal in $R$ contained in $J$. Alternatively, mono $(J)$ is the ideal generated by all monomials in $J$. There is an algorithm [20, Algorithm 4.4.2] for computing the generators of the monomial ideal $\operatorname{mono}(J)$.

Every weight vector $\mathbf{c} \in \mathbb{R}^{n}$ induces a partial order $\succeq$ on the monomials in $R$ via $\mathbf{x}^{\mathbf{u}} \succeq \mathbf{x}^{\mathbf{v}}$ if $\mathbf{c} \cdot \mathbf{u} \geq \mathbf{c} \cdot \mathbf{v}$. If $\mathbf{c} \in \mathbb{R}^{n}$ where 1 is the monomial of minimum $\mathbf{c}$-cost (that is, $\mathbf{c} \cdot \mathbf{u} \geq 0$ for every monomial $\mathbf{x}^{\mathbf{u}}$ ), then we can define initial terms and initial ideals. Given a polynomial $f=\sum_{\mathbf{u} \in \mathbb{N}^{n}} r_{\mathbf{u}} \mathbf{x}^{\mathbf{u}} \in I$ the initial term of $f$ with respect to $\mathbf{c}$, is denoted by $\operatorname{in}_{\mathbf{c}}(f)$, and equals the sum of all $r_{\mathbf{u}} \mathbf{x}^{\mathbf{u}}$ of $f$, where $\mathbf{c} \cdot \mathbf{u}$ is maximum. The initial ideal of $I$ with respect to $\mathbf{c}$ is defined as the ideal in $R$ generated by the initial terms of the polynomials in $I: \operatorname{in}_{\mathbf{c}}(I):=\left\langle\operatorname{in}_{\mathbf{c}}(f): f \in I\right\rangle$. A Gröbner basis of an ideal $I$ with respect to $\mathbf{c}$, is a finite collection of elements $g_{1}, \ldots, g_{s}$ in $I$ such that $\operatorname{in}_{\mathbf{c}}(I)=\left\langle\operatorname{in}_{\mathbf{c}}\left(g_{1}\right), \operatorname{in}_{\mathbf{c}}\left(g_{2}\right), \ldots, \operatorname{in}_{\mathbf{c}}\left(g_{s}\right)\right\rangle$. Every Gröbner basis is a generating set for the ideal $I$.

If $\operatorname{in}_{\mathbf{c}}(I)$ is a monomial ideal then a Gröbner basis is reduced if for every $i \neq j$, no term of $g_{i}$ is divisible by $\operatorname{in}_{\mathbf{c}}\left(g_{j}\right)$. The reduced Gröbner basis is unique. In this case, the set of monomials in $\operatorname{in}_{\mathbf{c}}(I)$ equal $\left\{\mathbf{x}^{\mathbf{u}}: \mathbf{u} \in U\right\}$ with $U:=D+\mathbb{N}^{n}$ where $D$ is the set of exponent vectors of the monomials $\operatorname{in}_{\mathbf{c}}\left(g_{1}\right), \operatorname{in}_{\mathbf{c}}\left(g_{2}\right), \ldots, \operatorname{in}_{\mathbf{c}}\left(g_{s}\right)$. Dickson's lemma states that sets of the form $D+\mathbb{N}^{n}$, where $D$ is arbitrary have only a finite number of minimal elements (with respect to coordinate wise inequalities). This is an alternative proof to Hilbert's result that every polynomial ideal is finitely generated. In this case, the Gröbner basis also provides a generalization of the Euclidean algorithm for polynomial rings with two or more variables called Buchberger's algorithm (see [8]). This algorithm solves the ideal membership problem: decide if a given polynomial is in an ideal or not. However, a Gröbner basis for an ideal can have many elements (relative to a minimal generating set for the ideal).

The toric ideal of $A$ is the ideal $I_{A}=\left\langle\mathbf{x}^{\mathbf{u}}-\mathbf{x}^{\mathbf{v}}: A \mathbf{u}=A \mathbf{v}, \mathbf{u}, \mathbf{v} \in \mathbb{N}^{n}\right\rangle$ and is called a binomial ideal since it is generated by polynomials having at most terms. Every reduced Gröbner basis of a toric ideal consists of binomials. A toric initial ideal is any initial ideal of a toric ideal. The following lemma is a natural connection between integer programming and toric initial ideals. 
Lemma 1. [20, Lemma 4.4.7] Let $A \in \mathbb{Z}^{d \times n}$ and $\mathbf{c} \in \mathbb{R}^{n}$. Then the monomial ideal mono( $\left.\operatorname{in}_{\mathbf{c}}\left(I_{A}\right)\right)$ is equal to

$$
\left\langle\mathbf{x}^{\omega}: \omega \in \mathbb{N}^{n} \text { is non-optimal solution for } \operatorname{IP}_{A, \mathbf{c}}(A \omega)\right\rangle \text {. }
$$

One direction of the proof of Lemma 1 is straightforward: let $\omega$ be a non-optimal solution, and $\omega^{\prime}$ an optimal solution to $\operatorname{IP}_{A, \mathbf{c}}(A \omega)$. Then $\mathbf{x}^{\omega}-\mathbf{x}^{\omega^{\prime}} \in I_{A}$ is a binomial with $\mathbf{x}^{\omega}$ as its initial term with respect to $\mathbf{c}$ and $\mathbf{x}^{\omega}$ is a monomial in mono(in $\left.\operatorname{in}_{\mathbf{c}}\left(I_{A}\right)\right)$. Our proof of the converse made essential use of Gröbner bases, and was longer, it is intuitive enough to be used without proof with the reference [20. Lemma 4.4.7] in the background.

A test set for the family of integer programs $\mathrm{IP}_{A, \mathbf{c}}$ is a collection of integer vectors $\left\{\mathbf{v}_{i}^{+}-\mathbf{v}_{i}^{-}: A \mathbf{v}_{i}^{+}=A \mathbf{v}_{i}^{-}, \mathbf{v}_{i}^{+}, \mathbf{v}_{i}^{-} \in \mathbb{N}^{n}, i=1, \ldots, s\right\}$ with the property that $\mathbf{u}$ is a feasible, non-optimal solution to $\mathrm{IP}_{A, \mathbf{c}}(\mathbf{b})$ if and only if there exists an $i, 1 \leq i \leq s$, such that $\mathbf{u}-\left(\mathbf{v}_{i}^{+}-\mathbf{v}_{i}^{-}\right) \geq \mathbf{0}$. We can now state our characterizations:

Theorem 1. Fix $A \in \mathbb{Z}^{d \times n}$ and $\mathbf{c} \in \mathbb{R}^{n}$, where $A$ is a Hilbert basis. The following statements are equivalent:

(i) The system $\mathbf{y} A \leq \mathbf{c}$ is $T D I$.

(ii) The subconfiguration $A_{\sigma}$ of $A$ is a Hilbert basis for every cell $\sigma$ in $\Delta_{\mathbf{c}}$.

(iii) There exists a test-set for $\mathrm{IP}_{A, \mathbf{c}}$ where all the positive coordinates are equal to 1 , the positive support consists of linearly independent columns, (and the negative support is a subset of a cell of $\left.\Delta_{\mathbf{c}}\right)$.

(iv) The monomial ideal $\left\langle\mathbf{x}^{\omega}: \omega \in \mathbb{N}^{n}\right.$ is not an optimal solution for $\left.\operatorname{IP}_{A, \mathbf{c}}(A \omega)\right\rangle$ has a square-free generating set.

(v) The monomial ideal generated by the set of monomials in $\operatorname{in}_{\mathbf{c}}\left(I_{A}\right)$ has a square-free generating set, that is, mono( $\left.\operatorname{in}_{\mathbf{c}}\left(I_{A}\right)\right)$ has a square-free generating set.

Proof. (i) is equivalent to (ii) : This is well-known from Schrijver's work, (see for instance [21]), but we provide the (very simple) proof here for the sake of completeness: Suppose the system $\mathbf{y} A \leq \mathbf{c}$ is TDI, and let $\sigma \in \Delta_{c}$. We show that $A_{\sigma}$ is a Hilbert basis. Let $\mathbf{b} \in \operatorname{cone}\left(A_{\sigma}\right)$. Since the optimal solutions for $\operatorname{LP}_{A, \mathbf{c}}(\mathbf{b})$ are exactly the non-negative combinations of the columns of $\mathcal{A}_{\sigma}$ with result $\mathbf{b}$, the TDI property means exactly that $\mathbf{b}$ can also be written as a non-negative integer combination of columns in $A_{\sigma}$, as claimed.

(ii) implies (iii) : Suppose (ii) holds true for $\Delta_{\mathbf{c}}$ of $A$. For every $\tau \subseteq[n]$ with $\tau$ not contained in any cell of $\Delta_{\mathbf{c}}$, let $\mathbf{b}_{\tau}:=\sum_{i \in \tau} a_{i}=A\left(\sum_{i \in \tau} \mathbf{e}_{i}\right)$. Since $\tau$ is not contained in any cell of $\Delta_{\mathbf{c}}$, there exists an optimal solution $\beta_{\tau}$ to $\operatorname{LP}_{A, \mathbf{c}}\left(\mathbf{b}_{\tau}\right)$ with $\mathbf{c} \cdot \beta_{\tau}<\mathbf{c} \cdot \sum_{i \in \tau} \mathbf{e}_{i}$. By the optimality of $\beta_{\tau}$ we must have $\operatorname{supp}\left(\beta_{\tau}\right) \subseteq \sigma$ for some $\sigma$ a cell of $\Delta_{\mathbf{c}}$. Since (ii) holds $A_{\sigma}$ is a Hilbert basis for every cell of $\Delta_{\mathbf{c}}$ and therefore $\beta_{\tau}$ can be chosen to be an integral vector. Let

$$
\mathcal{T}_{A, \mathbf{c}}:=\left\{\sum_{i \in \tau} \mathbf{e}_{i}-\beta_{\tau}: \tau \text { not contained in any cell of } \Delta_{\mathbf{c}}\right\}
$$


We claim that $\mathcal{T}_{A, \mathbf{c}}$ is a test set for $\operatorname{IP}_{A, \mathbf{c}}$. Suppose $\mathbf{b} \in \mathbb{Z}^{d}$ and $\omega \in \mathbb{N}^{n}$ satisfies $A \omega=\mathbf{b}$. That is, $\omega$ is a feasible solution to $\operatorname{LP}_{A, \mathbf{c}}(\mathbf{b})$.

If $\omega$ is an optimal solution then $\operatorname{supp}(\omega)$ is contained in a cell in $\Delta_{\mathbf{c}}$. Thus no vector in $\mathcal{T}_{A, \mathbf{c}}$ can be subtracted from it and remain in $\mathbb{N}^{n}$. Conversely, if $\omega$ is not an optimal solution to $\operatorname{LP}_{A, \mathbf{c}}(\mathbf{b})$ then $\operatorname{supp}(\omega) \subseteq[n]$ is not contained in any cell $\sigma$ of $\Delta_{\mathbf{c}}$ and so by basic linear programming there exists $\tau \subseteq \operatorname{supp}(\omega), A_{\tau}$ is linearly independent which is also not contained in any cell. $\omega-\left(\sum_{i \in \tau} \mathbf{e}_{i}-\beta_{\tau}\right) \geq \mathbf{0}$. Note that this integer vector is cheaper than $\omega$ with respect to $\mathbf{c}$.

(iii) implies (i): Suppose (iii) is true but for some $\mathbf{b} \in \operatorname{cone}(A)$ the linear program $\operatorname{LP}_{A, \mathbf{c}}(\mathbf{b})$ does not have an integer optimal solution. Let $\omega \in \mathbb{N}^{n}$ be the optimal solution to the integer program $\operatorname{IP}_{A, \mathbf{c}}(\mathbf{b})$ and let $\alpha / D$ be the optimal solution to $\operatorname{LP}_{A, \mathbf{c}}(\mathbf{b})$ where $\alpha \in \mathbb{N}^{n}$, and $D$ is a positive integer. Since $\operatorname{LP}_{A, \mathbf{c}}(\mathbf{b})$ does not have an integer optimal solution, we have $\mathbf{c} \cdot \alpha / D<\mathbf{c} \cdot \omega$. This also implies that $D \omega$ is not an optimal solution to $\operatorname{IP}_{A, \mathbf{c}}(D \mathbf{b})$.

By (iii) there exists a test set for solving the integer program $\operatorname{IP}_{A, \mathbf{c}}(D \mathbf{b})$ and so there exists a $\gamma^{+}{ }_{-} \gamma^{-}$with $\gamma^{+} \in\{0,1\}^{n}$ and $\gamma^{-} \in \mathbb{N}^{n}$ such that $\mathbf{c} \cdot\left(\gamma^{+}{ }_{-} \gamma^{-}\right)>0$ and with $D \omega-\left(\gamma^{+}-\gamma^{-}\right) \in \mathbb{N}^{n}$. Hence, $\operatorname{supp}\left(\gamma^{+}\right) \subseteq \operatorname{supp}(D \omega)=\operatorname{supp}(\omega)$. Since the value of all elements in $\gamma^{+}$is 0 or 1 then we also have $\omega \geq \gamma^{+}$, so $\omega-\left(\gamma^{+}-\gamma^{-}\right) \in \mathbb{N}^{n}$ is also a feasible solution to $\operatorname{IP}_{A, \mathbf{c}}(\mathbf{b})$ with $\mathbf{c} \cdot\left(\omega-\left(\gamma^{+}-\gamma^{-}\right)\right)<$ c $\cdot \omega$, in contradiction to the optimality of $\omega$.

(iii) is equivalent to (iv): Both (iii) and (iv) can be reformulated as follows: If $\omega \in \mathbb{N}^{n}$ is not an optimal solution to $\operatorname{LP}_{A, \mathbf{c}}(A \omega)$ then the vector $\omega^{\prime}$ defined as $\omega_{i}^{\prime}:=1$ if $i \in \operatorname{supp}(\omega)$ and 0 otherwise is also a non-optimal solution to $\operatorname{LP}_{A, \mathbf{c}}\left(A \omega^{\prime}\right)$.

(iv) is equivalent to (v): This is a special case of Lemma 1.

Recall that we defined $\mathbf{c} \in \mathbb{R}^{n}$ to be generic with the first of the following conditions, but the others are also equivalent to the definition [28]:

- The integer program $\operatorname{IP}_{A, \mathbf{c}}(\mathbf{b})$ has a unique optimal solution for all $\mathbf{b} \in \mathbb{N} \mathcal{A}$.

- The toric initial ideal $\operatorname{in}_{\mathbf{c}}\left(I_{A}\right)$ is a monomial ideal.

- There exists a Gröbner basis $\left\{\mathbf{x}^{\mathbf{u}_{1}^{+}}-\mathbf{x}^{\mathbf{u}_{1}^{-}}, \ldots, \mathbf{x}^{\mathbf{u}_{s}^{+}}-\mathbf{x}^{\mathbf{u}_{s}^{-}}\right\}$of $I_{A}$ with $\mathbf{c} \cdot \mathbf{u}_{i}^{+}>$ $\mathbf{c} \cdot \mathbf{u}_{i}^{-}$for each $i=1, \ldots, s$.

In the generic case, by Cramer's rule, (ii) is equivalent to $\Delta_{\mathbf{c}}$ being a unimodular triangulation which gives the following corollary.

Corollary 1. (Sturmfels) [26. Corollary 8.9] Let $A \in \mathbb{Z}^{d \times n}$ and let $c \in \mathbb{R}^{n}$ be generic with respect to $A$. Then $\Delta_{\mathbf{c}}$ is a unimodular triangulation if and only if the toric initial ideal $\operatorname{in}_{\mathbf{c}}\left(I_{A}\right)$ is generated by square-free monomials.

Still concerning generic $\mathbf{c}$ it is worth to note the following result of Conti and Traverso which provides another connection between integer linear programming and Gröbner bases. Here we think of an element $\mathbf{x}^{\mathbf{v}^{+}}-\mathbf{x}^{\mathbf{v}^{-}}$in the reduced Gröbner basis as a vector $\mathbf{v}^{+}-\mathbf{v}^{-}$. 
Proposition 1. (Conti-Traverso) [2] - see [29, Lemma 3] If $\operatorname{IP}_{A, \mathbf{c}}(\mathbf{b})$ has a unique optimal solution for every $\mathbf{b} \in \mathbb{N} A$ then the reduced Gröbner basis is a minimal test set for the family of integer programs $\mathrm{IP}_{A, \mathbf{c}}$.

This proposition means for us that in the generic case the following (vi) can be added to Theorem 1

(vi) The initial terms in the reduced Gröbner basis are square-free.

In particular, in the generic case of condition (iii) of Theorem 1 the unique inclusion wise minimal test set is defined by the reduced Gröbner basis, which, by (vi) has only square-free terms initial terms.

As is typically the case in combinatorial optimization, the cost vector $\mathbf{c}$ is not generic for $A$. Theorem 1 was found by a desire to generalize Sturmfels' theorem. In the rest of this section we study the limits of profiting from the advantages of the generic case by refinement. ¿From the implication "(ii) implies (i)" we immediately get the following:

Proposition 2. If $\mathbf{c}, \mathbf{c}^{\prime} \in \mathbb{R}^{n}$ are such that $\Delta_{\mathbf{c}^{\prime}}$ of $A$ is a refinement of $\Delta_{\mathbf{c}}$ of $A$, where $\Delta_{\mathbf{c}^{\prime}}$ is a unimodular triangulation of $A$, then $\mathbf{y} A \leq \mathbf{c}$ is TDI.

Clearly, the unimodular triangulation does not even need to be regular - a unimodular cover of the cells is actually enough as well for verifying - by Cramer's rule - that $A_{\sigma}$ is a Hilbert basis, and therefore (ii) holds. We are interested in the converse of Proposition 2, that is, the existence of such a $\mathbf{c}^{\prime}$ for every TDI system. In general such a converse does not hold. It is not even true that a Hilbert basis has a unimodular partition or a unimodular covering [1. This counterexample 1 inspires two important remarks. First, it cannot be expected that the equivalence of (i) and (v) can be reduced to Sturmfels' generic case, even though square-free generating sets exist for general TDI systems as well. Secondly, it should be appreciated that the converse of this remark does hold in the important set packing special case, as we will see in the next Section 3 .

\section{Set Packing}

Let a set packing problem be defined with a matrix $A$ and vector $\mathbf{c}$, and recall $\mathbf{c}:=(\mathbf{1}, \mathbf{0}) \in \mathbb{R}^{n}$, where the last $d$ entries of $\mathbf{c}$ are 0 . If the set packing polytope $Q_{\mathbf{c}}$ has integer vertices then the matrix $A$ and the polytope $Q_{\mathbf{c}}$ are said to be perfect. (We will not use the well-known equivalence of this definition with the integer values of optima: this will follow.) Lovász' (weak) perfect graph theorem [16] is equivalent to: the matrix $A$ defining a set packing polytope is perfect if and only if its first $(n-d)$ columns form the incidence vectors (indexed by the vertices) of the inclusion wise maximal complete subgraphs of a perfect graph.

A polyhedral proof of the perfect graph theorem can be split into two parts: Lovász' replication lemma [16] and Fulkerson's pluperfect graph theorem [12. The latter states roughly that a set packing polytope with integer vertices is described by a TDI system of linear inequalities. In this section we restate Fulkerson's result 
in a sharper form: there is a unimodular regular triangulation that refines the normal fan of any integral set packing polytope. We essentially repeat Fulkerson's proof, completing it with a part that shows unimodularity along the lines of the proof of [23, Theorem 3.1]. The following theorem contains the weak perfect graph theorem and endows it with an additional geometric feature. Denote the common optimal value of $\operatorname{LP}_{A, \mathbf{c}}(\mathbf{b})$ and $\mathrm{DP}_{A, \mathbf{c}}(\mathbf{b})$ by $\gamma_{\mathbf{c}}(\mathbf{b})$. Note that $\gamma_{\mathbf{c}}$ is a monotone increasing function in all of the coordinates.

Theorem 2. Let $Q_{\mathbf{c}}$ be a set packing polytope defined by $A$ and $\mathbf{c}$. Then there exists a vector $\varepsilon \in \mathbb{R}^{n}$ such that $\mathbf{c}^{\prime}:=(\mathbf{1}, \mathbf{0})+\varepsilon$ defines a regular triangulation $\Delta_{\mathbf{c}^{\prime}}$ refining $\Delta_{\mathbf{c}}$, and this triangulation is unimodular, if and only if $Q_{\mathbf{c}}$ is perfect.

We do not claim that the following proof of this theorem is novel. All essential ingredients except unimodularity are already included in the proof of Fulkerson's pluperfect graph theorem [12]. Cook, Fonlupt and Schrijver 4] and Chandrasekaran, Tamir [2] both exploited the fact that the greedy way of taking active rows leads to integer basic solutions in this case. The latter paper extensively used lexicographically best solutions, which is an important tool in linear programming theory, and this was used in observing the existence of a unimodular refinement of the normal fan in 23. This same lexicographic perturbation is accounted for by the vector $\varepsilon$ of Theorem 2, showing that the unimodular refinement is regular. This motivated the following problem, thus containing perfectness test:

Problem 1. 24] Given a $d \times n$ integer matrix $A$ and an $n$ dimensional integer vector $c$, decide in polynomial time whether the normal fan of $Q_{\mathbf{c}}$ consists only of unimodular cones. Equivalently, can it be decided in polynomial time that $Q_{\mathbf{c}}$ is non-degenerate, and the determinant of $A_{\sigma}$ is \pm 1 for all $\sigma \in \Delta_{\mathbf{c}}$.

Theorem 2 is a last step in a sharpening series of observations all having essentially the same proof. We begin similarly, with the proof of Fulkerson's pluperfect graph theorem which will indicate what the $\mathbf{c}^{\prime}$ of Theorem 2 should be, and then finish by showing that $\Delta_{\mathbf{c}^{\prime}}$ is a unimodular triangulation.

Assume that $A$ is a perfect matrix for the remainder of this section and that $\mathbf{c}=(\mathbf{1}, \mathbf{0})$ as before. For all $\mathbf{b} \in \mathbb{Z}^{d}$ and column index $i \in\{1, \ldots, n\}$ let

$$
\lambda_{\mathbf{c}, i}(\mathbf{b}):=\max \left\{x_{i}: \mathbf{x} \text { is an optimal solution of } \operatorname{LP}_{A, \mathbf{c}}(\mathbf{b})\right\} .
$$

That is, $\lambda_{\mathbf{c}, i}(\mathbf{b})$ is the largest value of $x_{i}$ such that $\mathbf{c} \cdot \mathbf{x}$ is minimum under $\mathbf{x} \in P_{\mathbf{b}}$.

An additional remark: if $\sigma$ is the minimal cell of $\Delta_{\mathbf{c}} \operatorname{such} \mathbf{b} \in \operatorname{cone}\left(A_{\sigma}\right)$, then $\mathbf{b}-\lambda_{\mathbf{c}, i}(\mathbf{b}) \mathbf{a}_{i} \in \operatorname{cone}\left(A_{\sigma^{\prime}}\right)$ where $\sigma^{\prime} \in \Delta_{\mathbf{c}}, \sigma^{\prime} \subseteq \sigma$ and the dimension of cone $\left(A_{\sigma^{\prime}}\right)$ is strictly smaller than that of cone $\left(A_{\sigma}\right)$. Furthermore, $\mathbf{b}-\lambda \mathbf{a}_{i} \notin \operatorname{cone}\left(A_{\sigma}\right)$ if $\lambda>\lambda_{\mathbf{c}, i}(\mathbf{b})$.

For all $\mathbf{b} \in \mathbb{Z}^{d}$ we show that $\lambda_{\mathbf{c}, i}(\mathbf{b})$ is an integer for every $i=1, \ldots, n$. This is the heart of Fulkerson's pluperfect graph theorem [12, Theorem 4.1]. We state it here in a way that is most useful for our needs: 
Lemma 2. Suppose $\gamma_{\mathbf{c}}(b) \in \mathbb{Z}$ for all $\mathbf{b} \in \mathbb{Z}^{d}$. If $\mathbf{x}$ is an optimal solution to $\mathrm{LP}_{A, \mathbf{c}}(\mathbf{b})$ with $x_{l} \neq 0$ for some $1 \leq l \leq n$, then there exists $\mathbf{x}^{*}$ also optimal for the same $\mathbf{b}$, such that $x_{l}^{*} \geq 1$.

Note that this lemma implies the integrality of $\lambda:=\lambda_{\mathbf{c}, l}(\mathbf{b})$ for all $l=1, \ldots, n$ : if $\lambda$ were not an integer then setting $\mathbf{b}^{\prime}:=\mathbf{b}-\lfloor\lambda\rfloor \mathbf{a}_{l}$ we have $\lambda_{\mathbf{c}, l}\left(\mathbf{b}^{\prime}\right)=\{\lambda\}$ where $0 \leq\{\lambda\}:=\lambda-\lfloor\lambda\rfloor<1$, contradicting Lemma 2 ,

Proof. Suppose $\mathbf{x} \in P_{\mathbf{b}}$ with $\mathbf{c} \cdot \mathbf{x}=\gamma(\mathbf{b})$ and $x_{l}>0$ for some $1 \leq l \leq n$. We have two cases: either $1 \leq l \leq n-d$ or $n-d+1 \leq l \leq n$.

If $n-d+1 \leq l \leq n$ then $\mathbf{a}_{l}=-\mathbf{e}_{l-(n-d)} \in \mathbb{R}^{d}$ and $c_{l}=0$. In this case, we have $\gamma_{\mathbf{c}}(\mathbf{b})=\gamma_{\mathbf{c}}\left(\mathbf{b}+x_{l} \mathbf{e}_{l-(n-d)}\right)$ because replacing $x_{l}$ by 0 in $\mathbf{x}$ we get a solution of the same objective value for the right hand side $\mathbf{b}+x_{l} \mathbf{e}_{l-(n-d)}$ which gives $\gamma_{\mathbf{c}}(\mathbf{b}) \geq \gamma_{\mathbf{c}}\left(\mathbf{b}+x_{l} \mathbf{e}_{l-(n-d)}\right)$. The reverse inequality follows from the (coordinatewise) monotonicity of $\gamma_{\mathbf{c}}$. But then

$$
\gamma_{\mathbf{c}}\left(\mathbf{b}+\mathbf{e}_{l-(n-d)}\right) \leq \gamma_{\mathbf{c}}\left(\mathbf{b}+x_{l} \mathbf{e}_{l-(n-d)}\right)+1-x_{l}=\gamma_{\mathbf{c}}(\mathbf{b})+1-x_{l},
$$

and since $\gamma_{\mathbf{c}}\left(\mathbf{b}+\mathbf{e}_{l-(n-d)}\right)$ is integer and $1-x_{l}<1$, we conclude that $\gamma_{\mathbf{c}}(\mathbf{b}+$ $\left.\mathbf{e}_{l-(n-d)}\right)=\gamma_{\mathbf{c}}(\mathbf{b})$.

So for any optimal $\mathbf{x}^{\prime} \in P_{\mathbf{b}+\mathbf{e}_{l-(n-d)}}$ where $\mathbf{c} \cdot \mathbf{x}^{\prime}=\gamma_{\mathbf{c}}(\mathbf{b})$, letting $\mathbf{x}^{*}:=$ $\mathbf{x}^{\prime}+\mathbf{e}_{l-(n-d)} \in P_{\mathbf{b}}$ we have $\mathbf{c} \cdot \mathbf{x}^{*} \leq \gamma_{\mathbf{c}}(\mathbf{b})$ and so $\mathbf{x}^{*}$ is optimal and $x_{l}^{*} \geq 1$.

On the other hand, suppose $1 \leq l \leq n-d$. By the monotonicity of $\gamma_{\mathbf{c}}$, and noting that replacing $x_{l}$ in $\mathbf{x}$ by 0 we get a point in $P_{\mathbf{b}-x_{l} \mathbf{a}_{l}}$. This point has objective value $\mathbf{c} \cdot \mathbf{x}-x_{l}<\mathbf{c} \cdot \mathbf{x}=\gamma_{\mathbf{c}}(\mathbf{b})$, and so we have

$$
\gamma\left(\mathbf{b}-\mathbf{a}_{l}\right) \leq \gamma\left(\mathbf{b}-x_{l} \mathbf{a}_{l}\right)<\gamma(\mathbf{b}) .
$$

Since the left and right hand sides are both integer values then $\gamma\left(\mathbf{b}-\mathbf{a}_{l}\right) \leq$ $\gamma(\mathbf{b})-1$. In other words, for any optimal $\mathbf{x}^{\prime} \in P_{\mathbf{b}-\mathbf{a}_{l}}$ we have $\mathbf{c} \cdot \mathbf{x}^{\prime} \leq \gamma_{\mathbf{c}}(\mathbf{b})-1$. Letting $\mathbf{x}^{*}:=\mathbf{x}^{\prime}+\mathbf{e}_{l} \in P_{\mathbf{b}}$ we get $\mathbf{c} \cdot \mathbf{x}^{*} \leq \gamma_{\mathbf{c}}(\mathbf{b})-1+1=\gamma_{\mathbf{c}}(\mathbf{b})$ with $x_{l}^{*} \geq 1$.

Let us know define the appropriate $\mathbf{c}^{\prime}$ for the theorem, depending only on $\mathbf{c}$. Define $\mathbf{c}^{\prime}:=\mathbf{c}+\varepsilon \in \mathbb{R}^{n}$ where $\varepsilon_{i}:=-\left(1 / n^{n+2}\right)^{i}$ for each $i=1, \ldots, n$. Note that the absolute value of the determinant of a $\{-1,0,1\}$-matrix cannot exceed $n^{n}$. It follows, by Cramer's rule, that the coefficients of linear dependencies between the columns of $A$ are at most $n^{n}$ in absolute value, and then the sum of absolute values of the coefficients between two solutions of an equation $A \mathbf{x}=\mathbf{b}$ for any $b \in \mathbb{R}^{n}$ can differ by at most a factor of $n^{n+2}$. After this observation two facts can be immediately checked (this is well-known from courses of linear programming):

(i) Any optimal solution to $\operatorname{LP}_{A, \mathbf{c}^{\prime}}(\mathbf{b})$ is also optimal for $\operatorname{LP}_{A, \mathbf{c}}(\mathbf{b})$.

(ii) If $\mathbf{x}^{\prime}$ and $\mathbf{x}^{\prime \prime}$ are both optimal solutions to $\operatorname{LP}_{A, \mathbf{c}}(\mathbf{b})$ then $\mathbf{x}^{\prime}$ is lexicographically bigger than $\mathrm{x}^{\prime \prime}$ (that is, the first non-zero coordinate of $\mathrm{x}^{\prime}-\mathrm{x}^{\prime \prime}$ is positive) if and only if $\mathbf{c}^{\prime} \cdot \mathbf{x}^{\prime}<\mathbf{c}^{\prime} \cdot \mathbf{x}^{\prime \prime}$.

Fact (i) means that $\Delta_{\mathbf{c}^{\prime}}$ refines $\Delta_{\mathbf{c}}$, and (ii) means that an optimal solution to $\operatorname{LP}_{A, \mathbf{c}^{\prime}}(\mathbf{b})$ is constructed by defining $\mathbf{b}^{0}:=\mathbf{b}$ and recursively

$$
x_{i}:=\lambda_{\mathbf{c}, i}\left(\mathbf{b}^{i-1}\right), \mathbf{b}^{i}:=\mathbf{b}^{i-1}-x_{i} \mathbf{a}_{i} .
$$


Furthermore, this optimum is unique and it follows that $\Delta_{\mathbf{c}^{\prime}}$ is a triangulation. We are now ready to prove Theorem 2

Proof of Theorem 2. The necessity of the condition is straightforward: each vertex $y \in Q_{\mathbf{c}}$ satisfies the linear equation of the form $y A_{\sigma^{\prime}}=1$, where $\sigma^{\prime}$ is a cell of $\Delta_{\mathbf{c}^{\prime}}, b \in \operatorname{cone}\left(A_{\sigma^{\prime}}\right) \subseteq \operatorname{cone}\left(A_{\sigma}\right), \sigma \in \Delta_{\mathbf{c}}$. Since the determinant of $A_{\sigma}$ is \pm 1 , by Cramer's rule, $y$ is integer.

Conversely, we will prove the assertion supposing only that $\gamma_{\mathbf{c}}(\mathbf{b})$ is integer for all $\mathbf{b} \in \mathbb{Z}^{d}$. (Note that then by the already proven easy direction we will have proved from this weaker statement that $Q_{\mathbf{c}}$ is perfect, as promised at the definition of perfectness.)

Without loss of generality, suppose that $\mathbf{b}$ cannot be generated by less than $d$ columns of $A$, that is, the minimal cell $\sigma$ of $\Delta_{\mathbf{c}}$ such that $\mathbf{b} \in \operatorname{cone}\left(A_{\sigma}\right)$ is a maximal cell of $\Delta_{\mathbf{c}}$. That is, $\operatorname{cone}\left(A_{\sigma}\right)$ is $d$-dimensional. Because of fact (i), an optimal solution to $\operatorname{LP}_{A, \mathbf{c}^{\prime}}(\mathbf{b})$ will have support in $\sigma$ and fact (ii) implies that such an optimal solution is constructed as follows:

Let $s_{1}:=\min \{i: i \in \sigma\}$ and $x_{s_{1}}:=\lambda_{\mathbf{c}, s_{1}}(\mathbf{b})$. Recursively, for $j=2, \ldots, d$ let $s_{j}$ be the smallest element in $\sigma$ indexing a column of $A$ on the minimal face of cone $\left(A_{\sigma}\right)$ containing

$$
\mathbf{b}-\sum_{i=1}^{j-1} x_{s_{i}} \mathbf{a}_{s_{i}} .
$$

Since $b$ is in the interior of $\operatorname{cone}\left(A_{\sigma}\right)$ then $x_{s_{i}}>0$ for each $i=1, \ldots, d$, and by Lemma 2 , these $d x_{s_{i}}$ 's are integer. Moreover, since the dimension of cone $\left(A_{\sigma \backslash\left\{s_{1}, \ldots, s_{i}\right\}}\right)$ is strictly decreasing as $i=2, \ldots, d$ progresses then

$$
\mathbf{b}-\sum_{i=1}^{d} x_{s_{i}} \mathbf{a}_{s_{i}}=0
$$

and, setting $U:=\left\{s_{1}, \ldots, s_{d}\right\} \subseteq \sigma$, we have the columns of $A_{U}$ are linearly independent. Note that $U$ is a cell of $\Delta_{\mathbf{c}^{\prime}}$ and every maximal cell of $\Delta_{\mathbf{c}^{\prime}}$ arises in this fashion. We show that the matrix $A_{U}$ has determinant \pm 1 .

Suppose not. Then the inverse of the matrix $A_{U}$ is non-integer, and from the matrix equation $\left(A_{U}\right)^{-1} A_{U}=$ id we see that there exists a unit vector $\mathbf{e}_{j} \in \mathbb{R}^{d}$ which is a noninteger combination of columns in $A_{U}$ :

$$
\sum_{i=1}^{d} x_{s_{i}} \mathbf{a}_{s_{i}}=\mathbf{e}_{j} .
$$

For $\alpha \in \mathbb{R}$ let $\{\alpha\}:=\alpha-\lfloor\alpha\rfloor$, and define:

$$
\sum_{i=1}^{d}\left\{x_{s_{i}}\right\} \mathbf{a}_{s_{i}}=: \mathbf{z}
$$

Clearly, $\mathbf{z} \in \operatorname{cone}\left(A_{U}\right)$ and furthermore $\mathbf{z} \in \mathbb{Z}^{d}$ since it differs from $\mathbf{e}_{\mathbf{j}}$ by an integer combination of the columns of $A_{U}$. So Lemma 2 can be applied to $\mathbf{b}:=\mathbf{z}$ : 
letting $l:=\min \left\{i:\left\{x_{s_{i}}\right\} \neq 0\right\}$ we see that $\lambda_{\mathbf{c}, s_{l}}(\mathbf{z})<1$ contradicting Lemma 2 Hence both $A_{U}$ and $\left(A_{U}\right)^{-1}$ are integer, their determinant is \pm 1 ; since $A_{U}$ was an arbitrary maximal cell of $\Delta_{\mathbf{c}^{\prime}}$, we conclude that $\Delta_{\mathbf{c}^{\prime}}$ is unimodular.

The argument concerning the inverse matrix replaces the use of parallelepipeds (compare with [23, proof of Theorem 3.1]) that we wanted to avoid here to stay in elementary terms.

Note that all the numbers in the definition of $\mathbf{c}^{\prime}$ are at most $n^{n^{2}}$, so they have a polynomial number of digits: the perturbed problem has polynomial size in terms of the original one, reducing perfectness test to Problem 1

\section{Computation}

In this section we wish to give an idea of how the results presented in this work lead to practical algorithms. There are three essentially different approaches.

A first, general, elementary algorithm can be based on Theorem 1, or more precisely on the proof of its Corollary ??. Indeed, the procedure described in this corollary is a general algorithm for testing the TDI property in $O\left(n^{d}\right)$ time. If $d$ is fixed, it is a polynomial algorithm. This is very recent and has not yet been implemented.

The equivalences of (i) and (v) in Theorem 11along with an algorithm 20, Algorithm 4.4.2] for computing the generators of the monomial ideal mono $\left(\operatorname{in}_{\mathbf{c}}\left(I_{A}\right)\right)$ permit us to detect TDI using algebraic methods: the generators are square-free if and only if the system $\mathbf{y} A \leq \mathbf{c}$ is TDI.

This algorithm works for all cost vectors, be they generic or non-generic, but it is not yet implemented and our suspition is that mono( $\left.\operatorname{in}_{\mathbf{c}}\left(I_{A}\right)\right)$ could be rather difficult to compute in the non-generic case. However, in the generic case, $\operatorname{in}_{\mathbf{c}}\left(I_{A}\right)$ is already a monomial ideal and can be attained in practice. In addition, even if $\mathbf{c}$ is non-generic, it may have a generic perturbation yielding a unimodular triangulation and then the toric initial ideals can be studied with respect to the perturbed vector. Computing the toric initial ideal may be far easier than investigating the unimodularity of the corresponding triangulation.

Let us have a look at one example of an $A$ and $\mathbf{c}$ coming from a set packing problem. A more efficient way of treating the data is at hand in the generic case. Then we can use the computationally well studied reduced Gröbner bases according to Proposition 1 .

The perfect graph in Figure 1 with 21 maximal cliques on 20 vertices was constructed by Padberg in [18]. The matrix $A$ is a $(20 \times 41)$-matrix and the toric ideal $I_{A}$ lives in the polynomial ring $\mathbf{k}\left[a, \ldots, u, v_{1}, \ldots, v_{20}\right]$ where $a, \ldots, u$ correspond to the maximal cliques of $G$ (the first 21 columns of $A$ ) and where $v_{1}, \ldots, v_{20}$ correspond to the vertices of $G$ (the ordered columns of $-I_{20}$, the last 20 columns of $A$ ) as before.

The toric initial ideal with an appropriate perturbation has 61 elements, all of which are square-free. The computation was carried out in Macaulay 2 [14 (in less than 1 second) and its implementation can be seen in [17, Appendix A]. 


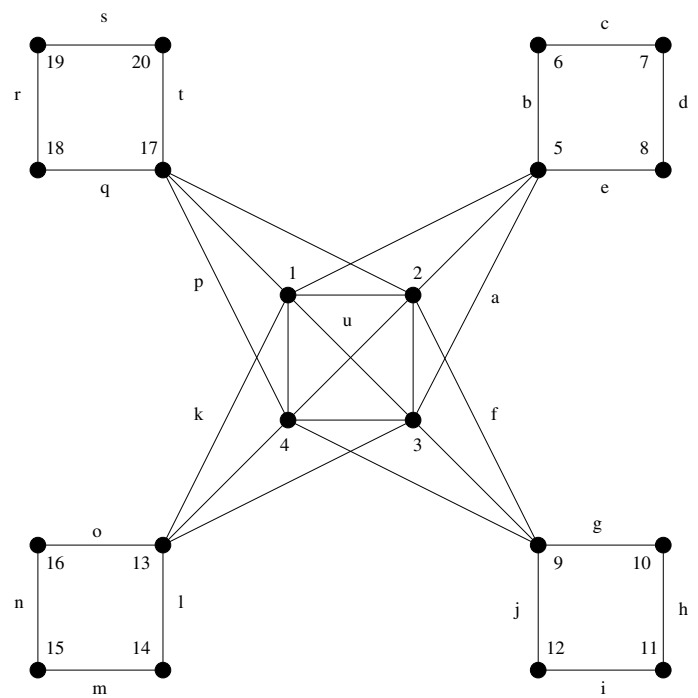

Fig. 1. Padberg's graph G with 21 maximal cliques on 20 vertices

However, we could (equivalently) have asked if a well-defined triangulation refining $\Delta_{\mathbf{c}}$, was a unimodular triangulation. This is a far more exhausting task than computing the monomial toric initial ideal. Because of the bijection between the cells of $\Delta_{\mathbf{c}}$ and the vertices of $Q_{\mathbf{c}}$, using PORTA 9] we computed that $Q_{\mathbf{c}}$ had precisely 5901 vertices. Next, using TOPCOM [19] a number of these 5901 cells are each refined into many pieces by the refinement. To confirm TDI, the determinant indexed by each of the many refined cells would have to be computed.

\section{Acknowledgments}

The authors wish to thank Rekha Thomas for her valuable input and suggestions. Some work related to the results of this article, including the computational experimentation, can be found in the first author's Ph.D. dissertation at the University of Washington. Thanks are also due to our colleagues who developed the computational packages Macaulay 2 and TOPCOM.

\section{References}

1. W. Bruns, J. Gubeladze, Normality and Covering Properties of Affine Semigroups, manuscript.

2. R. Chandrasekaran, A. Tamir, On the integrality of an extreme solution to pluperfect graph and balanced systems, Oper. Res. Let., 3, (1984), 215-218.

3. P. Conti, C. Traverso, Buchberger algorithm and integer programming, Applied algebra, algebraic algorithms and error-correcting codes, Lecture Notes in Comput. Sci., 539, Springer, Berlin, 1991 
4. W. Cook, J. Fonlupt, A. Schrijver, An integer analogue of Carathéodory's theorem. J. Combin. Theory(B), 40, (1986), 63-70.

5. W. Cook, L. Lovász, A. Schrijver, A polynomial-time test for total dual integrality in fixed dimension, Mathematical Programming Study 22, (1984), 64-69.

6. G. CornuÉJols, Combinatorial optimization: packing and covering, CBMS-NSF regional conference series in applied mathematics, 74 SIAM, 2001.

7. M. Chudnovsky, G. Cornuésols, L. Xinming, K. Vušković, Recognizing Berge graphs. Combinatorica, 25, (2005), no. 2, 143-186.

8. D. Cox, J. Little, D. O'Shea, Ideals, varieties and algorithms, 2nd edition, Springer-Verlag, NY, 1996.

9. T. Christof, A. LÖBel, PORTA (POlyhedron Representation Transformation Algorithm), available from http://www.zib.de/Optimization/Software/Porta/.

10. V. ChVÁtal, On certain polytopes associated with graphs Journal of Comb. Theory,(B), 18, (1975), 138-154.

11. A. Gerards, A. Sebő, Total dual integrality implies local strong unimodularity, Mathematical Programming, 38 (1987), 69-73.

12. D. R. Fulkerson, Anti-blocking polyhedra, Journal of Comb. Theory,(B), 12, (1972), 50-71.

13. J. Graver, On the foundations of linear and integer programming I, Math. Programming 8, (1975), 207-226.

14. D. Grayson, M. Stillman, Macaulay 2, a software system for research in algebraic geometry, available from http://www.math.uiuc.edu/Macaulay2/.

15. S. Hoşten, R.R. Thomas, Gomory integer programs, Mathematical Program$\operatorname{ming}(\mathrm{B}), \mathbf{9 6},(2003), 271-292$.

16. L. LovÁsz, Normal hypergraphs and the perfect graph conjecture, Discrete Mathematics, 2, (1972), 253-267.

17. E. O'SheA, Toric algebra and the weak perfect graph theorem, Ph.D. dissertation, University of Washington, 2006.

18. M. Padberg, Perfect zero-one matrices, Math. Programming 6 (1974), 180-196.

19. J. RAmbau, TOPCOM (Triangulations Of Point Configurations and Oriented Matroids), available from http://www.uni-bayreuth.de/departments/ wirtschaftsmathematik/rambau/TOPCOM/

20. M. Saito, B. Sturmfels, N. Takayama, Gröbner deformations of hypergeometric differential equations, Algorithms and Computation in Mathematics, 6 Springer-Verlag, Berlin, 2000.

21. A. SchriJver, Theory of linear and integer programming, Wiley, 1986.

22. A. SchriJver, Combinatorial optimization: polyhedra and efficiency, Algorithms and Combinatorics 24, Springer, 2003.

23. A. SEвö, Hilbert bases, Caratheodory's theorem and combinatorial optimization, Integer Programming and Combinatorial Optimization (eds: R. Kannan, W. Pulleyblank) Mathematical Programming Society, University of Waterloo Press, Waterloo, 1990.

24. A. SEBö, Problem A.6, "TDI Matrices", in Open Problems (Workshop on "The Perfect Graph Conjecture", (2002), http://www.aimath.org/pastworkshops/perfectgraph.html.

25. B. Sturmfels, R. Weismantel, G. M. Ziegler, Gröbner bases of lattices, corner polyhedra, and integer programming, Beiträge Algebra Geom., 36, (1995), 281-298.

26. B. Sturmfels, Gröbner bases and convex polytopes, University Lecture Series 8, American Mathematical Society, Providence, RI, 1996. 
27. B. Sturmfels, Algebraic recipes for integer programming, AMS Shortcourse: Trends in Optimization (eds: S. Hoşten, J. Lee, R.R. Thomas) Proceedings of Symposia in Applied Mathematics, 61, American Mathematical Society, Providence, RI, 2004.

28. B. Sturmfels and R. R. Thomas, Variations of cost functions in integer programming, Math. Programming 77, (1997), 357-387.

29. R. R. Thomas, Algebraic methods in integer programming, Encyclopedia of Optimization (eds: C. Floudas and P. Pardalos), Kluwer Academic Publishers, Dordrecht, 2001 